\title{
Random walks on finite lattice tubes
}

\author{
B.I. Henry* \\ Department of Applied Mathematics, \\ School of Mathematics, \\ University of New South Wales, \\ Sydney NSW 2052, Australia \\ M.T. Batchelol ${ }^{\dagger}$ \\ Department of Theoretical Physics, \\ Research School of Physical Sciences and Engineering, \\ and Centre for Mathematics and its Applications, \\ Mathematical Sciences Institute, \\ Australian National University Canberra ACT 0200, Australia
}

(Dated: today) 


\begin{abstract}
Exact results are obtained for random walks on finite lattice tubes with a single source and absorbing lattice sites at the ends. Explicit formulae are derived for the absorption probabilities at the ends and for the expectations that a random walk will visit a particular lattice site before being absorbed. Results are obtained for lattice tubes of arbitrary size and each of the regular lattice types; square, triangular and honeycomb. The results include an adjustable parameter to model the effects of strain, such as surface curvature, on the surface diffusion. Results for the triangular lattice tubes and the honeycomb lattice tubes model diffusion of adatoms on single walled zig-zag carbon nano-tubes with open ends.
\end{abstract}

PACS numbers: 02.50.Ey, 05.40Fb

Keywords: random walk, lattice, diffusion, difference equation, nano-tube

*Electronic address: b.henry@unsw.edu.au

†Electronic address: murrayb@maths.anu.edu.au 


\section{INTRODUCTION}

The problem of random walks on finite lattices is fundamental to the theory of stochastic processes [1] and has numerous applications including potential theory [2], electrical networks 3], atomic surface diffusion [4] and diffusion on biological membranes [5]. A classic problem in this area, which was posed by Courant et al. [6] in 1928, concerns random walks on finite planar lattices with a single source and absorbing boundaries. The exact solution for this problem on the square lattice was derived in 1940 7]. The exact solution on the triangular lattice was only obtained recently [8, 9] after having been considered intractable 10]. Other variants of the problem on the square lattice have also been solved exactly [10, 11, 12].

In this paper we consider random walks from a single source on a finite lattice which incorporates periodic boundary conditions in one direction but absorbing boundary conditions in the other. Diffusion thus occurs on a surface with the topology of a lattice tube with absorbing sites at the ends. We present explicit results for each of the three regular lattice types; square, triangular and honeycomb. Our results include a bias parameter that can be adjusted away from unity to model different random walk probabilities in the cyclic direction around the tube compared with the axial direction along the tube. This parameter can be adjusted to model the effect of surface curvature, and other types of strain, on the surface diffusion.

Our derivations generalize the approach developed by McCrea and Whipple 7] for planar square lattice random walks with absorbing boundaries. This is straightforward in the case of the square lattice tube however special care has to be exercised in an appropriate choice of co-ordinates in the case of the triangular lattice tube and the honeycomb lattice tube.

There are two important motivations for our study. One of the motivations is to add to the rather small class of exactly solvable random walk lattice problems with absorbing boundaries, since it is still the case that: "Explicit solutions are known in only a few cases" 13]. The second motivation is that the mathematics of discrete lattice diffusion problems may find applications in the recently realized laboratory assembly of lattice nanostructures (see for example, [14]). For example our problem of random walks on the triangular lattice tube and the honeycomb lattice tube represent models for adatom diffusion on single walled zig-zag carbon nano-tubes [15] with open ends - the random walks on the triangular lattice tube model adatom diffusion across carbon-carbon bonds and the random walks on the 
honeycomb lattice tube model diffusion along the carbon-carbon bonds. The diffusion of carbon adatoms along the carbon-carbon bonds of a carbon nanotube plays a vital role in stabilizing and maintaining the open edge growth of nanotubes [16, 17]. Our exact results complement related results for diffusion on carbon nano-tubes based on i) enumeration of random walks up to a set length [18] and ii) microcanonical molecular dynamics simulations [19].

The remainder of the paper is divided into separate sections for square lattice tubes, triangular lattice tubes, honeycomb lattice tubes, and a section containing an example and discussion.

\section{SQUARE LATTICE TUBES}

Consider the standard square lattice co-ordinates $(p, q)$ representing the intersections of equidistant vertical straight lines and equidistant horizontal straight lines. The expectation that a random walk starting from a site $(a, b)$ visits a site $(p, q)$, distinct from $(a, b)$, before being absorbed at a finite boundary site is given by the homogeneous partial difference equation

$$
\begin{array}{r}
F(p, q)=\frac{1}{2+2 \eta}[F(p+1, q)+F(p-1, q) \\
+\eta F(p, q+1)+\eta F(p, q-1)] .
\end{array}
$$

The parameter $\eta$ allows for different probabilities for walks around the tube compared with walks along the tube. To accommodate the source term at $(a, b)$ we construct separate solutions; $F_{I}(p, q)$ for $q \leq b$ and $F_{I I}(p, q)$ for $q \geq b$. The expectation that a random walk starting from $(a, b)$ visits a site $(p, b)$, not necessarily distinct from $(a, b)$, before being absorbed at a finite boundary is then given by the inhomogeneous partial difference equation

$$
\begin{aligned}
F_{I}(p, b)=\delta_{p, a}+ & \frac{1}{2+2 \eta}\left[F_{I}(p+1, b)+F_{I}(p-1, b)\right. \\
& \left.+\eta F_{I}(p, b-1)+\eta F_{I I}(p, b+1)\right] .
\end{aligned}
$$

The above difference equations are to be solved with periodic boundary conditions in the $p$ co-ordinates,

$$
F(p, q)=F(p+m+1, q)
$$


absorbing boundary conditions in the $q$ co-ordinates,

$$
\begin{aligned}
F_{I}(p, 0) & =0, \\
F_{I I}(p, n+1) & =0,
\end{aligned}
$$

and matching conditions at $q=b$, ie.,

$$
F_{I}(p, b)=F_{I I}(p, b) .
$$

The method of solving inhomogeneous linear partial difference boundary value problems as above consists of two parts [7, 9, 10, 13]. First obtain the general separation of variables solution to the homogeneous problem, then find an appropriate linear combination of such solutions to satisfy the boundary conditions and the inhomogeneous problem. A major difficulty in these problems can be the identification of a lattice co-ordinate system with a separation of variables solution that can be matched with the boundary conditions [9].

The homogeneous field equations for the square lattice, Eq.(1), admit the separable solution

$$
F(p, q)=P(p) Q(q)
$$

where

$$
\begin{aligned}
P(p+1)+(\lambda-(2+2 \eta)) P(p)+P(p-1) & =0, \\
Q(q+1)-\frac{\lambda}{\eta} Q(q)+Q(q-1) & =0,
\end{aligned}
$$

and $\lambda$ is the separation constant. These separated equations have general solutions

$$
\begin{aligned}
& P(p)=A \mu^{p}+B \mu^{-p}, \\
& Q(q)=C \nu^{q}+D \nu^{-q},
\end{aligned}
$$

where

$$
\begin{aligned}
& \mu=\frac{2+2 \eta-\lambda}{2}+\frac{\sqrt{(2+2 \eta-\lambda)^{2}-4}}{2}, \\
& \nu=\frac{\lambda}{2 \eta}+\frac{\sqrt{\frac{\lambda^{2}}{\eta^{2}}-4}}{2} .
\end{aligned}
$$

If $\lambda=2 \eta$ then the solutions are no longer provided by Eqs. (10) and (11). In this case we have the solutions

$$
\begin{aligned}
& P(p)=\hat{A}+\hat{B} p \\
& Q(q)=\hat{C}+\hat{D} q .
\end{aligned}
$$


The special solutions in Eqs.(14) and (15) do not appear in the planar lattice problems because they cannot satisfy absorbing boundary conditions in both $p$ and $q$ co-ordinates.

With suitable linear combinations of the above solutions, Eqs.(10),(11),(14), (15), we find that the general solutions to the homogeneous problem that also satisfy the boundary conditions, Eqs.(3), (4), and the matching condition, Eq.([6), can be written in the form

$$
\begin{aligned}
& F_{I}(p, q)=c q(b-n-1)+\sum_{k=1}^{m}\left(a_{k} e^{i \alpha_{k} p}+b_{k} e^{-i \alpha_{k} p}\right) \\
& \times \sinh \left(\beta_{k} q\right) \sinh \left[\beta_{k}(b-n-1)\right] \\
&\left.F_{I I}(p, q)=c b(q-n-1)\right)+\sum_{k=1}^{m}\left(a_{k} e^{i \alpha_{k} p}+b_{k} e^{-i \alpha_{k} p}\right) \\
& \times \sinh \left[\beta_{k}(q-n-1)\right] \sinh \left(\beta_{k} b\right)
\end{aligned}
$$

where

$$
\alpha_{k}=\frac{2 \pi k}{m+1}
$$

and

$$
2+2 \eta=2 \eta \cosh \beta_{k}+2 \cos \alpha_{k}
$$

The constants $c, a_{k}$ and $b_{k}$ are now determined by the requirement that the solutions satisfy the inhomogeneous equation, Eq.(21). This step is facilitated using the identity in Eq.(19) together with the identity in Eq.(A.1) and the Kronecker delta identity

$$
\sum_{k=0}^{m} e^{2 \pi i(p-a) k /(m+1)}=(m+1) \delta_{p, a}
$$

re-arranged as

$$
\begin{array}{r}
\delta_{p, a}=\frac{1}{m+1}+\frac{1}{2(m+1)}\left(\sum_{k=1}^{m} e^{i \alpha_{k} p} e^{-i \alpha_{k} a}\right. \\
\left.+\sum_{k=1}^{m} e^{-i \alpha_{k} p} e^{i \alpha_{k} a}\right) .
\end{array}
$$

We thereby obtain the following solutions for the expectations that a random walk will visit a site $(p, q)$ before being absorbed at an end site:

$$
\begin{aligned}
F_{I}(p, q)= & \frac{(2+2 \eta) q(n+1-b)}{\eta(n+1)(m+1)} \\
& +\frac{2+2 \eta}{\eta(m+1)} \sum_{k=1}^{m} \cos \left[\alpha_{k}(p-a)\right] \\
& \times \frac{\sinh \left[\beta_{k}(n+1-b)\right] \sinh \left(\beta_{k} q\right)}{\sinh \left(\beta_{k}\right) \sinh \left[\beta_{k}(n+1)\right]},
\end{aligned}
$$




$$
\begin{aligned}
F_{I I}(p, q)= & \frac{(2+2 \eta) b(n+1-q)}{\eta(n+1)(m+1)} \\
& +\frac{2+2 \eta}{\eta(m+1)} \sum_{k=1}^{m} \cos \left[\alpha_{k}(p-a)\right] \\
& \times \frac{\sinh \left[\beta_{k}(n+1-q)\right] \sinh \left(\beta_{k} b\right)}{\sinh \left(\beta_{k}\right) \sinh \left[\beta_{k}(n+1)\right]},
\end{aligned}
$$

with $\beta_{k}$ dependent on $k$ through Eqs.(18),(19).

The absorption probabilities $G(p, q)$ are readily evaluated from

$$
\begin{aligned}
G(p, 0) & =\frac{\eta}{2+2 \eta} F_{I}(p, 1), \\
G(p, n+1) & =\frac{\eta}{2+2 \eta} F_{I I}(p, n) .
\end{aligned}
$$

It is a simple matter to show that

$$
\sum_{p=0}^{m} G(p, 0)=\left(\frac{n+1-b}{n+1}\right)
$$

and

$$
\sum_{p=0}^{m} G(p, n+1)=\left(\frac{b}{n+1}\right)
$$

so that

$$
\sum_{p=0}^{m}[G(p, 0)+G(p, n+1)]=1 .
$$

\section{TRIANGULAR LATTICE TUBES}

To obtain the solution for triangular lattice tubes we consider the lattice co-ordinates $(p, q)$ shown in Figure 1. This co-ordinate system describes two independent triangular lattice systems, only one of which can be accessed by a random walk from a single point source. In Figure 1 the sites that are accessible from the source site at $(a, b)$ are indicated by filled circles. The nearest neighbour sites to the source are highlighted by open circles in this figure. The lattice sites that are not accessible from the source are referred to as the zero mesh [10]. The same co-ordinate system has been used to find an approximate solution to the planar triangular lattice problem with absorbing boundaries [10] however a different zig-zag co-ordinate system was required to find the exact solution [9]. In the case of the planar triangular lattice problem the co-ordinate system in Figure 1 allows the leakage of random walks from sites at $p=1$ and $p=m$ to sites at $p=-1$ and $p=m+2$ respectively, 


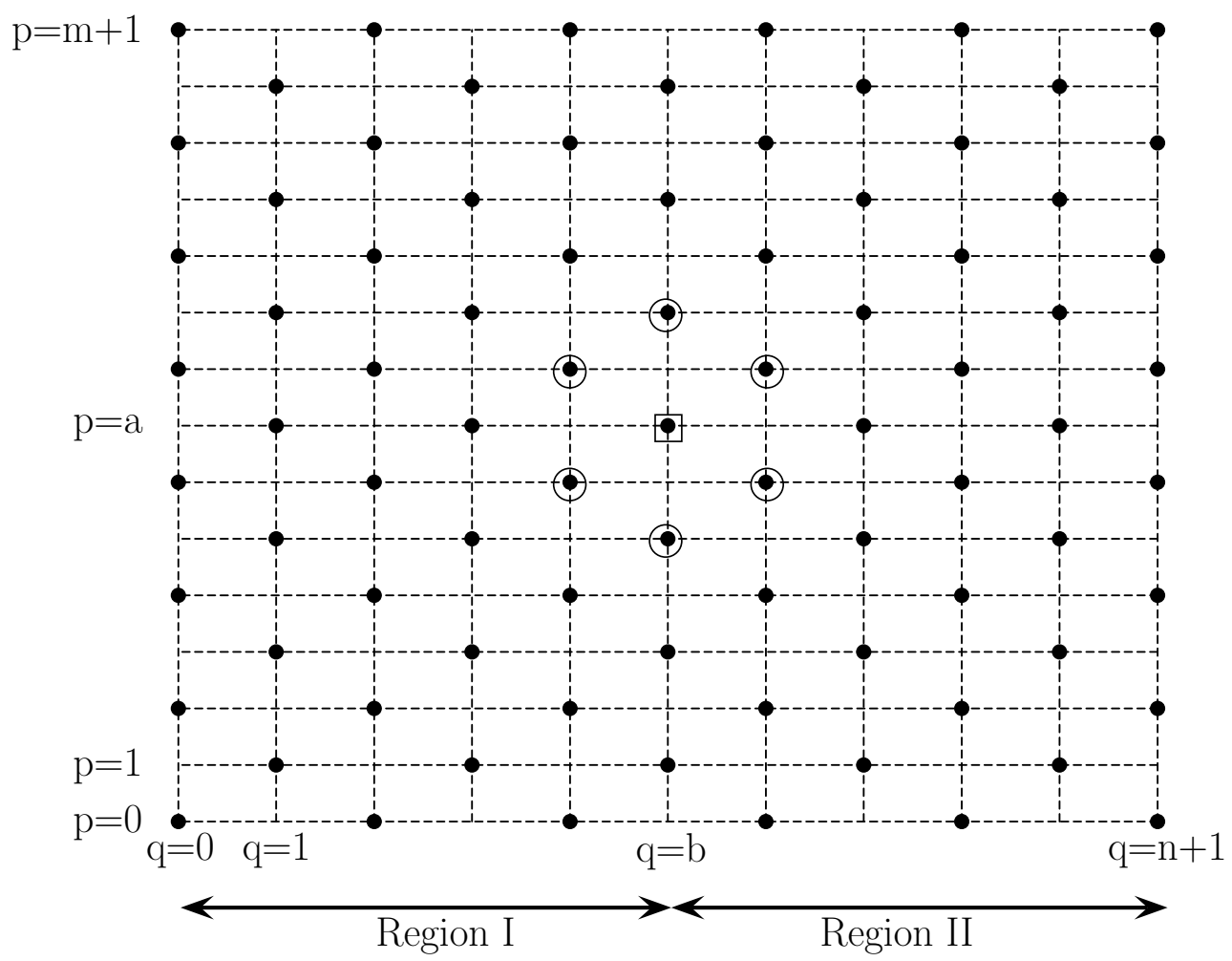

FIG. 1: Triangular lattice with $(p, q)$ co-ordinates as used in section III.

which are outside the absorbing boundaries at $p=0$ and $p=m+1$. In the triangular lattice tube this leakage is prevented because the $p$ co-ordinate is cyclic. Note that $m+1$ must be an even integer to permit periodic boundaries in the $p$ direction.

With the co-ordinate system shown in Figure 1 the problem on the triangular lattice tube is described by the homogeneous equation, for $(p, q) \neq(a, b)$,

$$
\begin{array}{r}
F(p, q)=\frac{1}{2+4 \eta}[F(p+2, q)+F(p-2, q) \\
+\eta F(p+1, q+1)+\eta F(p+1, q-1) \\
+\eta F(p-1, q+1)+\eta F(p-1, q-1)]
\end{array}
$$

the inhomogeneous equation, for $q=b$,

$$
\begin{aligned}
F_{I}(p, b)= & \delta_{p, a}+\frac{1}{2+4 \eta}\left[F_{I}(p+2, b)+F_{I}(p-2, b)\right. \\
& +\eta F_{I I}(p+1, b+1)+\eta F_{I}(p+1, b-1) \\
& \left.+\eta F_{I I}(p-1, b+1)+\eta F_{I}(p-1, b-1)\right]
\end{aligned}
$$


and the boundary conditions, Eqs.(3), (44), (6)

The homogeneous equation separates as

$$
\begin{aligned}
P(p+2)+\lambda P(p+1)-(2+4 \eta) P(p) & \\
+\lambda P(p-1)+P(p-2) & =0, \\
Q(q+1)-\frac{\lambda}{\eta} Q(q)+Q(q-1) & =0,
\end{aligned}
$$

with solutions

$$
\begin{aligned}
& P(p)=A e^{i \alpha p}+B^{-i \alpha p}+C e^{i \beta p}+D e^{-i \beta p}, \\
& Q(q)=E e^{\gamma q}+F e^{-\gamma q}
\end{aligned}
$$

where

$$
\begin{aligned}
& \alpha=\cos ^{-1}\left(-\frac{\lambda}{4}+\frac{1}{4} \sqrt{\lambda^{2}+16+16 \eta}\right) \\
& \beta=\cos ^{-1}\left(-\frac{\lambda}{4}-\frac{1}{4} \sqrt{\lambda^{2}+16+16 \eta}\right), \\
& \gamma=\cosh ^{-1}\left(\frac{\lambda}{2 \eta}\right) .
\end{aligned}
$$

The general solution to the homogeneous problem on the triangular lattice that satisfies all the boundary conditions, Eqs.(3),(41),(6), can be written as

$$
\begin{aligned}
F_{I}(p, q)= & c q(b-n-1) \\
+ & d q(b-n-1) \cos [\pi(p-a)] \cos [\pi(q-b)] \\
+ & \sum_{k=1}^{m^{\prime}}\left(a_{k} e^{i \alpha_{k} p}+b_{k} e^{-i \alpha_{k} p}\right) \\
& \times \sinh \left(\gamma_{k} q\right) \sinh \left[\gamma_{k}(b-n-1)\right], \\
F_{I I}(p, q)= & c b(q-n-1) \\
+ & d b(q-n-1) \cos [\pi(p-a)] \cos [\pi(q-b)] \\
+ & \sum_{k=1}^{m}\left(a_{k} e^{i \alpha_{k} p}+b_{k} e^{-i \alpha_{k} p}\right) \\
& \times \sinh \left[\gamma_{k}(q-n-1)\right] \sinh \left(\gamma_{k} b\right),
\end{aligned}
$$

where the prime on the sum has been used to indicate that the sum does not include the value $k=(m+1) / 2$ and

$$
\alpha_{k}=\frac{2 \pi k}{m+1}
$$


with

$$
2 \eta \cosh \gamma_{k} \cos \alpha_{k}=1+2 \eta-\cos 2 \alpha_{k}
$$

The homogeneous solutions of the form

$$
(\hat{A}+\hat{B} q) \cos [\pi(p-a)] \cos [\pi(q-b)]
$$

in Eqs.(35), (36), are important in two fundamental ways. First they replace the null solutions at $k=(m+1) / 2$ in the representation

$$
\left(a_{k} e^{i \alpha_{k} p}+b_{k} e^{-i \alpha_{k} p}\right) \sinh \left(\gamma_{k} q\right) \sinh \left[\gamma_{k}(b-n-1)\right]
$$

and secondly they allow the appropriate zero mesh solution for lattice co-ordinates that cannot be accessed by a source at $(a, b)$.

The constants $c, d, a_{k}, b_{k}$ are found by substituting the homogeneous solutions, Eqs.(35),(36), into the inhomogeneous equation, Eq.(27). Using the identity in Eq.(38) together with the idendity in Eq.A.1 we first obtain the intermediate result

$$
\begin{array}{r}
-(2+4 \eta) \delta_{p, a}=2 \eta c(n+1)+2 \eta d(n+1) \cos [\pi(p-a)] \\
+2 \eta \sum_{k=1}^{m^{\prime}}\left(a_{k} e^{i \alpha_{k} p}+b_{k} e^{-i \alpha_{k} p}\right) \cos \alpha_{k} \sinh \gamma_{k} \sinh \left[\gamma_{k}(n+1)\right]
\end{array} .
$$

The final result is then found by expanding the Kronecker delta as

$$
\begin{array}{r}
\delta_{p, a}=\frac{1}{m+1}+\frac{1}{m+1} \cos [\pi(p-a)] \\
+\frac{1}{2(m+1)}\left(\sum_{k=1}^{m^{\prime}} e^{i \alpha_{k} p} e^{-i \alpha_{k} a}+e^{-i \alpha_{k} p} e^{i \alpha_{k} a}\right) .
\end{array}
$$

Thus we obtain

$$
\begin{aligned}
F_{I}(p, q)= & \frac{(1+2 \eta) q(n+1-b)}{\eta(n+1)(m+1)} \\
& \times(1+\cos [\pi(p-a)] \cos [\pi(q-b)]) \\
& +\frac{1+2 \eta}{\eta(m+1)} \sum_{k=1}^{m} \cos \left[\alpha_{k}(p-a)\right] \\
& \times \frac{\sinh \left[\gamma_{k}(n+1-b)\right] \sinh \left(\gamma_{k} q\right)}{\cos \alpha_{k} \sinh \gamma_{k} \sinh \left[\gamma_{k}(n+1)\right]},
\end{aligned}
$$




$$
\begin{aligned}
F_{I I}(p, q)= & \frac{(1+2 \eta) b(n+1-q)}{\eta(n+1)(m+1)} \\
& \times(1+\cos [\pi(p-a)] \cos [\pi(q-b)]) \\
& +\frac{1+2 \eta}{\eta(m+1)} \sum_{k=1}^{m^{\prime}} \cos \left[\alpha_{k}(p-a)\right] \\
& \times \frac{\sinh \left[\gamma_{k}(n+1-q)\right] \sinh \left(\gamma_{k} b\right)}{\cos \alpha_{k} \sinh \gamma_{k} \sinh \left[\gamma_{k}(n+1)\right]}
\end{aligned}
$$

where $\alpha_{k}$ and $\gamma_{k}$ depend on $k$ through Eqs.(37),(38). Our results in Eqs.(42), (433) hold for

arbitrary $m$ except $m+1=0(\bmod 4)$ where singularities occur for $k=\frac{m+1}{4}, \frac{3(m+1)}{4}$. The absorption probabilities $G(p, q)$ are given by

$$
\begin{gathered}
G(p, 0)=\frac{\eta}{2+4 \eta}\left(F_{I}(p+1,1)+F_{I}(p-1,1)\right), \\
G(p, n+1)=\frac{\eta}{2+4 \eta}\left(F_{I I}(p+1, n)+F_{I I}(p-1, n)\right),
\end{gathered}
$$

The absorption probabilities at the ends of the tube are thus

$$
\sum_{p=0}^{m} G(p, 0)=\left(\frac{n+1-b}{n+1}\right)
$$

and

$$
\sum_{p=0}^{m} G(p, n+1)=\left(\frac{b}{n+1}\right)
$$

with

$$
\sum_{p=0}^{m}[G(p, 0)+G(p, n+1)]=1 .
$$

\section{HONEYCOMB LATTICE TUBES}

Here we consider a co-ordinate system in which we label the vertices of the honeycomb lattice by the intersection points $(p, q)$ of horizontal straight lines $p=0,1,2, \ldots, m+1$ and vertical zig-zag lines $q=0,1,2, \ldots, n+1$ (see Figure 2). The expectation that a random walk visits an interior site $(p, q)$, distinct from the starting site $(a, b)$, is given by the coupled homogeneous difference equations:

$$
\begin{array}{cc}
F(p, q)=\frac{1}{2+\eta} \quad[\eta \hat{F}(p, q+1)+\hat{F}(p+1, q) \\
+\hat{F}(p-1, q)], \\
\hat{F}(p, q)=\frac{1}{2+\eta} \quad \begin{array}{c}
{[\eta F(p, q-1)+F(p+1, q)} \\
+F(p-1, q)] .
\end{array}
\end{array}
$$




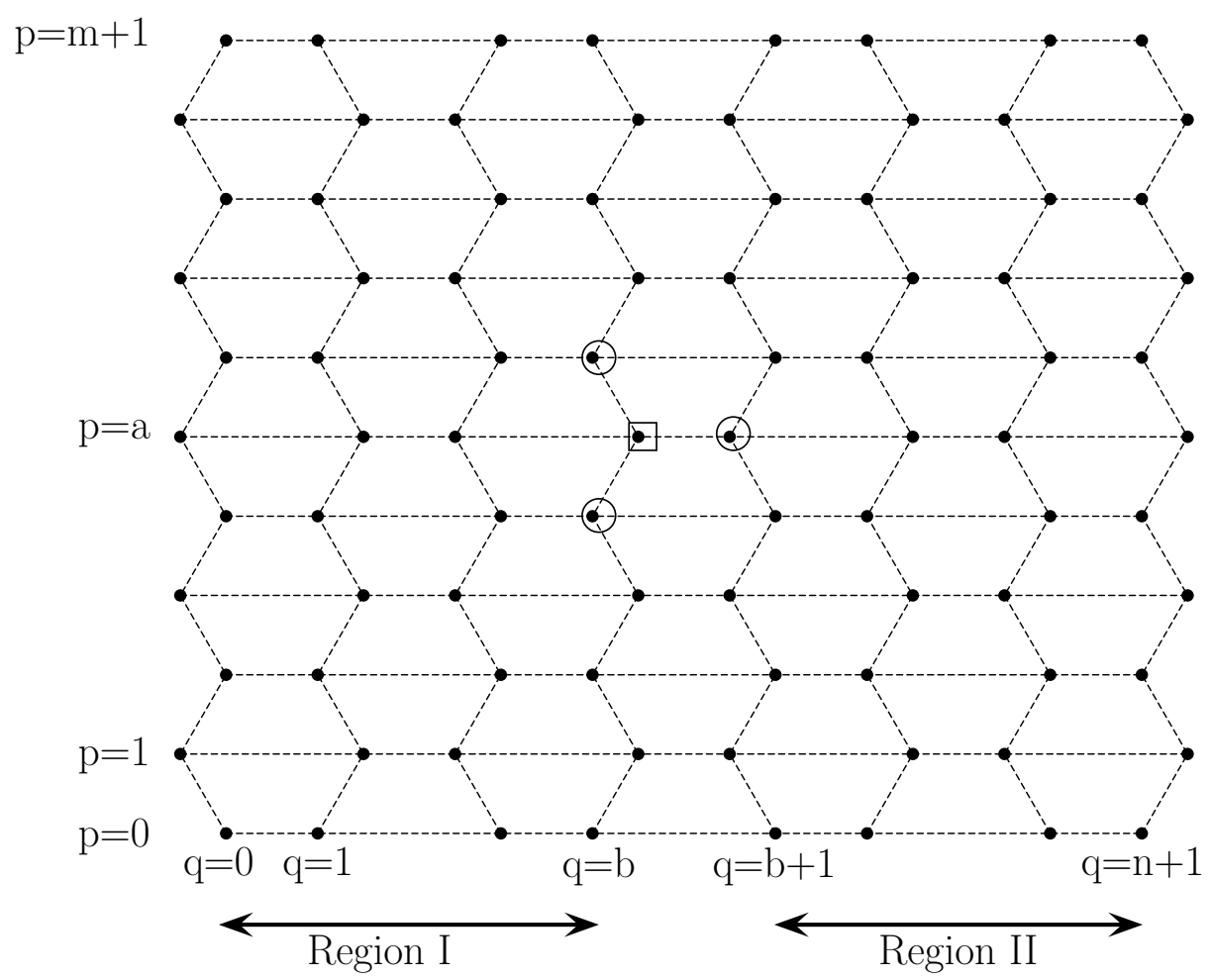

FIG. 2: Honeycomb lattice with $(p, q)$ co-ordinates as used in section IV.

Here $F(p, q)$ is the expectation at sites $(p, q)$ with nearest neighbours on the right at $(p, q+1)$ and $\hat{F}(p, q)$ is the expectation at sites $(p, q)$ with nearest neighbours on the left at $(p, q-1)$. We will refer to these distinct symmetry sites as $\vdash$ sites and $\dashv$ sites respectively. The appeal of this particular choice of $(p, q)$ co-ordinates is that the difference equations for the distinct symmetry sites can be decoupled into separable equations for each. Indeed both $F(p, q)$ and $\hat{F}(p, q)$ satisfy the same homogeneous triangular lattice equation as Eq.(26) except near the absorbing boundaries. This relationship between random walks on the honeycomb lattice and random walks on the triangular lattice is illustrated in Figure 3. In this figure the site at $(p, q)$ is shown as a $\vdash$ site. The nearest neighbour triangular lattice sites to the site at $(p, q)$ are of the same $\vdash$ symmetry type and co-ordinate labels for the honeycomb lattice and the triangular lattice match at all $\vdash$ sites. A similar matching occurs at $\dashv$ sites (as can be seen by inverting Figure 3). The nearest neighbours on the triangular lattice are next nearest neighbours on the honeycomb lattice and the probability of a random walk from 


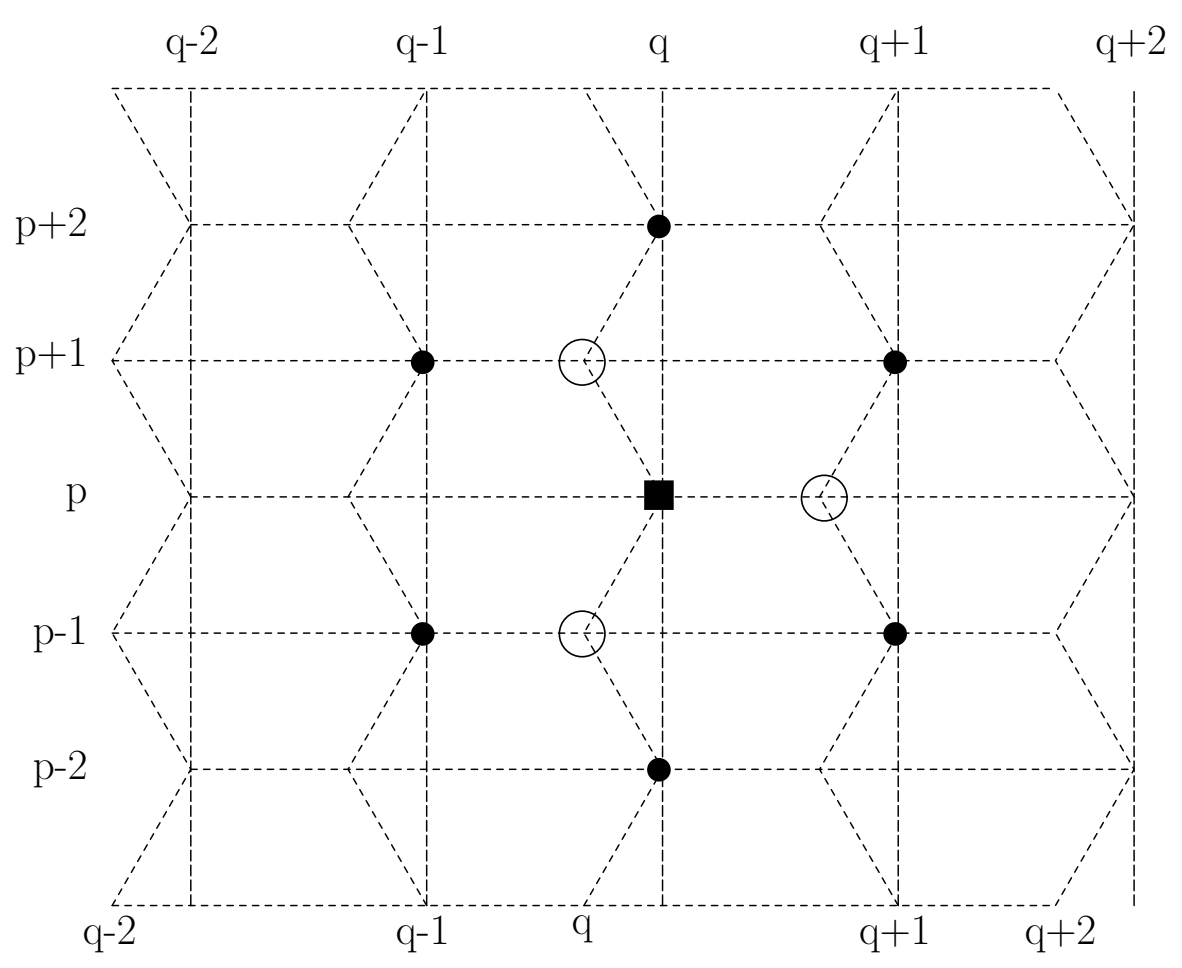

FIG. 3: Honeycomb lattice showing a site at $(p, q)$ (filled box) surrounded by nearest neighbours on a honeycomb lattice (large circles) and nearest neighbours on a triangular lattice (small filled circles). The $q$ labels at the bottom of the figure refer to the honeycomb lattice and those at the top refer to the triangular lattice.

$(p, q)$ to one of these sites is the same for random walks on the triangular lattice and the honeycomb lattice.

The triangular lattice equation fails for $F(p, n)$ due to the absorbing boundary condition $\hat{F}(p, n+1)=0$ and similarly the triangular lattice equation fails for $\hat{F}(p, 1)$ due to the absorbing boundary condition $F(p, 0)=0$. To circumvent these boundary problems we use the homogeneous triangular lattice equation solutions for $F(p, q)$ in the region $q \leq b$ (region I) and we use the homogeneous triangular lattice equation solutions for $\hat{F}(p, q)$ in the region 
$q \geq b+1$ (region II). Thus we have homogeneous solutions of the form

$$
\begin{aligned}
F_{I}(p, q)= & c q+d q \cos [\pi(p-a)] \cos [\pi(q-b)] \\
& +\sum_{k=1}^{m^{\prime}}\left(a_{k} e^{i \alpha_{k} p}+b_{k} e^{-i \alpha_{k} p}\right) \sinh \left(\gamma_{k} q\right), \\
\hat{F}_{I I}(p, q)= & \hat{c}(q-n-1) \\
& +\hat{d}(q-n-1) \cos [\pi(p-a)] \cos [\pi(q-b)] \\
& +\sum_{k=1}^{m^{\prime}}\left(\hat{a}_{k} e^{i \alpha_{k} p}+\hat{b}_{k} e^{-i \alpha_{k} p}\right) \sinh \left[\gamma_{k}(q-n-1)\right] .
\end{aligned}
$$

These homogeneous solutions satisfy the periodic boundary conditions as well as the absorbing boundary conditions $\hat{F}_{I I}(p, n+1)=0$ and $F_{I}(p, 0)=0$.

We now consider a single point source at a type $\vdash$ symmetry site. Thus we have the inhomogeneous problem

$$
\begin{aligned}
F_{I}(p, b)= & \delta_{p, a}+\frac{1}{2+\eta}\left[\eta \hat{F}_{I I}(p, b+1)\right. \\
& \left.+\hat{F}_{I}(p+1, b)+\hat{F}_{I}(p-1, b)\right] \\
\hat{F}_{I I}(p, b+1)= & \frac{1}{2+\eta}\left[\eta F_{I}(p, b)+F_{I I}(p+1, b+1)\right. \\
& \left.+F_{I I}(p-1, b+1)\right] .
\end{aligned}
$$

At this stage we do not have general solutions for $\hat{F}_{I}(p, q)$ and $F_{I I}(p, q)$ and so we use Eqs.(46), (47) to write the inhomogeneous problem in the form

$$
\begin{aligned}
F_{I}(p, b)= & {\left[(2+\eta)^{2} \delta_{p, a}+(2+\eta) \eta \hat{F}_{I I}(p, b+1)\right.} \\
+ & \eta F_{I}(p+1, b-1)+F_{I}(p+2, b) \\
+ & \left.\eta F_{I}(p-1, b-1)+F_{I}(p-2, b)\right] \\
& /\left[(2+\eta)^{2}-2\right] \\
\hat{F}_{I I}(p, b+1) & =\left[(2+\eta) \eta F_{I}(p, b)\right. \\
& +\eta \hat{F}_{I I}(p+1, b+2)+\hat{F}_{I I}(p+2, b+1) \\
& \left.+\eta \hat{F}_{I I}(p-1, b+2)+\hat{F}_{I I}(p-2, b+1)\right] \\
& /\left[(2+\eta)^{2}-2\right] .
\end{aligned}
$$


The unknown constants $c, d, a_{k}, b_{k}, \hat{c}, \hat{d}, \hat{a}_{k}, \hat{b}_{k}$ can now be obtained by substituting the homogeneous solutions, Eqs.(48), (49) and the Kronecker delta identity, Eq.(41), into the inhomogeneous equations Eqs.(52),(153) and equating linearly independent functions of $p$. The algebraic manipulations are simplified using the identities in Eqs. (A.2), (A.3).

The resulting expressions for the expectation values are

$$
\begin{aligned}
F_{I}(p, q)= & \frac{(2+\eta)^{2}}{2 \eta(m+1)}\left(1-\frac{(\eta+2) b}{(\eta+2) n+2}\right) q(1+\cos [\pi(p-a)] \cos [\pi(q-b)]) \\
+ & \frac{(2+\eta)^{2}}{2 \eta(m+1)} \sum_{k=1}^{m^{\prime}}\left\{\cos \left[\alpha_{k}(p-a)\right] \sinh \left(\gamma_{k} q\right)\right. \\
\times & {\left.\left[\left(\eta+4 \cosh \left(\gamma_{k}\right) \cos \left(\alpha_{k}\right)\right) \sinh \left[\gamma_{k}(b-n)\right]-2 \cos \alpha_{k} \sinh \left[\gamma_{k}(b+1-n)\right]\right]\right\} } \\
/ & \left\{\left(\eta \cosh \gamma_{k} \cos \alpha_{k}-1-\eta\right)\left(\cosh \left[\gamma_{k}(n-2)\right]-\cosh \left(\gamma_{k} n\right)\right)\right. \\
- & \left.\left(\eta+4 \cosh \gamma_{k} \cos \alpha_{k}\right) \cos \alpha_{k} \sinh \gamma_{k} \sinh \left(\gamma_{k} n\right)\right\} \\
\hat{F}_{I I}(p, q) & =\frac{(2+\eta)^{3} b(n+1-q)}{2(\eta n+2 n+2) \eta(m+1)}(1-\cos [\pi(p-a)] \cos [\pi(q-b)]) \\
& +\frac{(2+\eta)^{3}}{2 \eta(m+1)} \sum_{k=1}^{m}\left\{\cos \left[\alpha_{k}(p-a)\right] \sinh \left[\gamma_{k}(q-n-1)\right] \sinh \left(\gamma_{k} b\right)\right\} \\
& /\left\{\left(\eta \cosh \gamma_{k} \cos \alpha_{k}-1-\eta\right)\left(\cosh \left[\gamma_{k}(n-2)\right]-\cosh \left(\gamma_{k} n\right)\right)\right. \\
& \left.-\left(\eta+4 \cosh \gamma_{k} \cos \alpha_{k}\right) \cos \alpha_{k} \sinh \gamma_{k} \sinh \left(\gamma_{k} n\right)\right\}
\end{aligned}
$$

The expectation values at type $\dashv$ sites in region I and $\vdash$ sites in region II can now be obtained by substituting the solutions from Eq.(54) into Eq.(147) and the solutions from Eq.(55) into Eq.(46) respectively. The results are

$$
\begin{aligned}
\hat{F}_{I}(p, q) & =\frac{(2+\eta)}{2 \eta(m+1)}\left(1-\frac{(\eta+2) b}{(\eta+2) n+2}\right)((\eta+2) q-\eta)(1-\cos [\pi(p-a)] \cos [\pi(q-b)]) \\
& +\frac{(2+\eta)}{2 \eta(m+1)} \sum_{k=1}^{m^{\prime}}\left\{\cos \left[\alpha_{k}(p-a)\right]\left[\eta \sinh \left[\gamma_{k}(q-1)\right]+2 \cos \left(\alpha_{k}\right) \sinh \left(\gamma_{k} q\right)\right]\right. \\
& \left.\times\left[\left(\eta+4 \cosh \left(\gamma_{k}\right) \cos \left(\alpha_{k}\right)\right) \sinh \left[\gamma_{k}(b-n)\right]-2 \cos \alpha_{k} \sinh \left[\gamma_{k}(b+1-n)\right]\right]\right\} \\
& /\left\{\left(\eta \cosh \gamma_{k} \cos \alpha_{k}-1-\eta\right)\left(\cosh \left[\gamma_{k}(n-2)\right]-\cosh \left(\gamma_{k} n\right)\right)\right. \\
& \left.-\left(\eta+4 \cosh \gamma_{k} \cos \alpha_{k}\right) \cos \alpha_{k} \sinh \gamma_{k} \sinh \left(\gamma_{k} n\right)\right\}
\end{aligned}
$$




$$
\begin{aligned}
F_{I I}(p, q) & =\frac{(2+\eta)^{2} b[(\eta+2)(n-q)+2]}{2((\eta+2) n+2) \eta(m+1)}(1+\cos [\pi(p-a)] \cos [\pi(q-b)]) \\
& +\frac{(2+\eta)^{2}}{2 \eta(m+1)} \sum_{k=1}^{m^{\prime}}\left\{\cos \left[\alpha_{k}(p-a)\right] \sinh \left(\gamma_{k} b\right)\right. \\
& \left.\times\left(\eta \sinh \left[\gamma_{k}(q-n)\right]+2 \cos \left(\alpha_{k}\right) \sinh \left[\gamma_{k}(q-n-1)\right]\right)\right\} \\
& /\left\{\left(\eta \cosh \gamma_{k} \cos \alpha_{k}-1-\eta\right)\left(\cosh \left[\gamma_{k}(n-2)\right]-\cosh \left(\gamma_{k} n\right)\right)\right. \\
& \left.-\left(\eta+4 \cosh \gamma_{k} \cos \alpha_{k}\right) \cos \alpha_{k} \sinh \gamma_{k} \sinh \left(\gamma_{k} n\right)\right\}
\end{aligned}
$$

The absorption probabilities are defined by

$$
\begin{aligned}
\hat{G}(p, 0) & =0, \\
G(p, 0) & =\frac{\eta}{2+\eta} \hat{F}_{I}(p, 1), \\
\hat{G}(p, n+1) & =\frac{\eta}{2+\eta} F_{I I}(p, n), \\
G(p, n+1) & =0 .
\end{aligned}
$$

The total absorption probabilities at the ends of the tubes are thus

$$
\sum_{p=0}^{m} G(p, 0)=1-\frac{(\eta+2) b}{(\eta+2) n+2}
$$

and

$$
\sum_{p=0}^{m} G(p, n+1)=\frac{(\eta+2) b}{(\eta+2) n+2} .
$$

Note that the absorption probablilities in this case are functions of the bias parameter $\eta$. The square lattice tube and the triangular lattice tube are symmetric with respect to left/right walks along the axial direction and thus the probabilities for absorption at the ends depend only on the initial distance from the ends at which particles are released. The honeycomb lattice is not symmetric with respect to left/right walks along the axial direction. As a consequence the absorption probabilities at the ends of the tube depend on both the initial distance from the ends (which also determines the symmetry type, $\vdash$ or $\dashv$, of the initial lattice site) and the axial bias parameter.

\section{EXAMPLE AND DISCUSSION}

In this paper we have derived exact formulae for the expectations that a random walk starting at a lattice point $(a, b)$ will visit a lattice site $(p, q)$ on a lattice tube with absorbing 
lattice sites on the ends. The formulae for square lattice tubes, Eqs.(22),(23), triangular lattice tubes, Eqs.(42),(43), and honeycomb lattice cubes, Eqs.(154),(155),(156),(157) allow us to readily compute the expectation values for tubes of any specified size and arbitrary starting points. Moreover each of these solutions contains an adjustable parameter $\eta$ that can be adjusted away from unity to model different random walk probabilities in the cyclic direction around the tube compared with the axial direction along the tube.

As an example we consider the case of a honeycomb lattice tube with $m=17, n=29$ and three values of $\eta$; i) $\eta=1$, ii) $\eta=1 / 100$, and iii) $\eta=100$. We have taken the source to be centrally located at $a=9, b=15$ in each case. The expectation values at each of the lattice co-ordinates have been plotted in Figure 4. As might be anticipated, for small values of the axial bias parameter, $\eta \ll 1$, diffusion along the tube axis is very slow; the random walk cycles around the tube many times (several hundred times for $\eta=1 / 100$ ) before finally

being absorbed at one of the open ends. For large values of the axial bias parameter, $\eta \gg 1$, we might first anticipate rapid diffusion along the tube axis however the tower like plot in Figure 4(c) reveals that this is not the case. The random walk becomes trapped locally near the source as it moves back and forward between the source site and the nearest neighbour to the source. This effect is indeed a simple consequence of the honeycomb lattice geometry which has only one nearest neighbour along the axis direction.

A further interesting calculation is the steady state profile for expectation values along the lattice tube after summation over $p$. The profile is piecewise linear with a linear increase from $q=1$ up to $q=b$ followed by a linear decrease from $q=b$ to $q=n$. The slope of the linear portions is dependent on the parameter $\eta$. Explicit expressions for this slope as a function of $\eta$ can be readily evaluated from the formulae for the expectation values given in Eqs (54)-(57). For example for $q \leq b$ we have

$$
\begin{aligned}
E_{I}(q) & =\sum_{p=0}^{m} F_{I}(p, q)+\hat{F}_{I}(p, q) \\
& =\frac{(\eta+2)^{2}}{\eta}\left(1-\frac{(n+2) b}{(\eta+2) n+2}\right) q-\frac{(\eta+2)}{2}\left(1-\frac{(\eta+2) b}{(\eta+2) n+2}\right),
\end{aligned}
$$

It is clear from this equation that the slope diverges as $\eta \rightarrow 0$ and as $\eta \rightarrow \infty$. For the example considered here with $n=29$ and $b=15$ we have

$$
E_{I}(q)=\frac{(7 \eta+15)(\eta+2)(2(\eta+2) q-\eta)}{\eta(60+29 \eta)}
$$



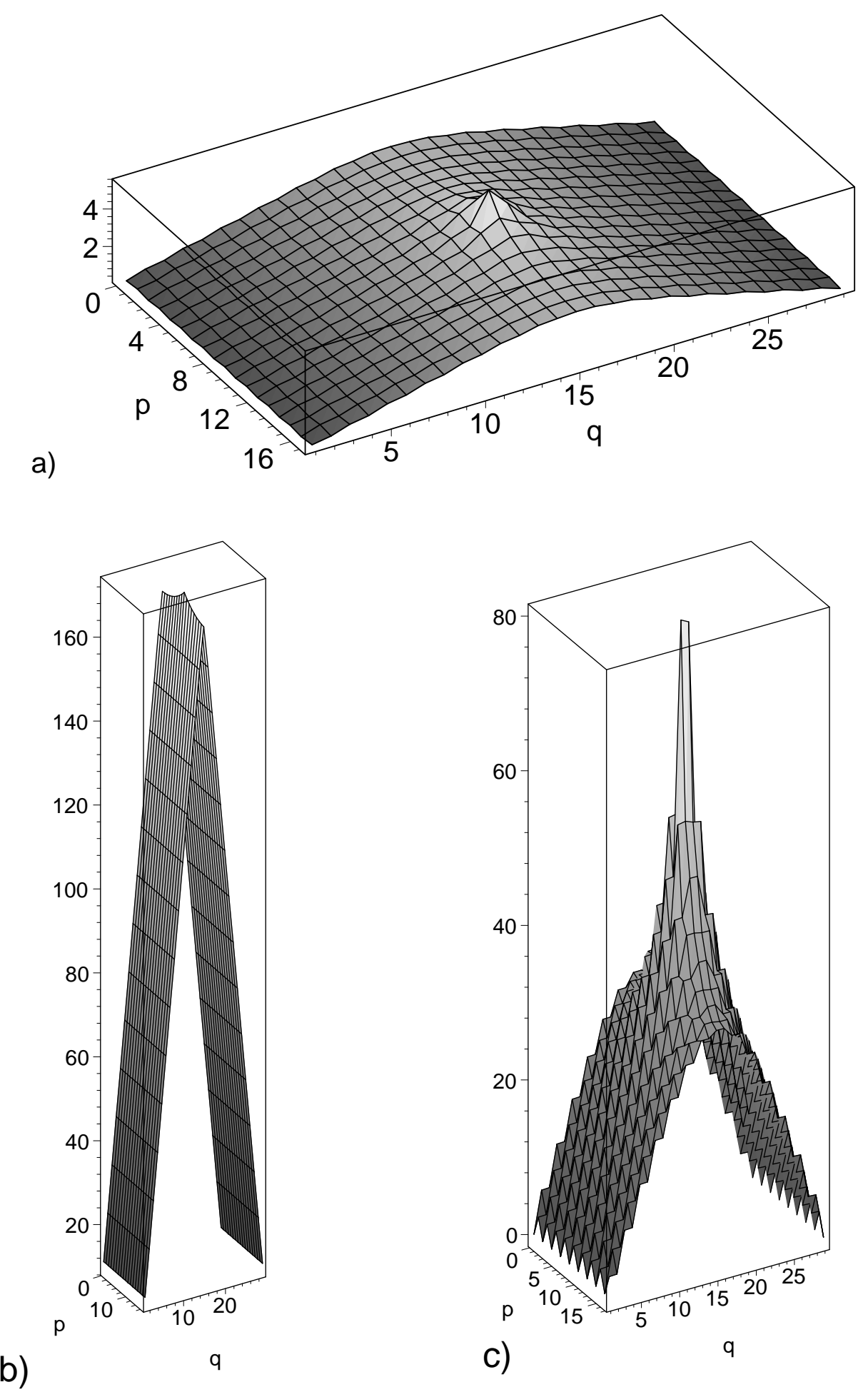

FIG. 4: Expectation values for a random walk on a honeycomb tube for three different values of the axial bias; a) $\eta=1$, b) $\eta=0.01$, c) $\eta=100$.

and the slope is a minimum at $\eta \approx 2.035$.

The geometry of the honeycomb lattice tube that we have considered in this paper is 
equivalent to that of a single walled zig-zag carbon nanotube with open ends. The above example corresponds to the $(9,0)$ nanotube in the standard notation [15]. The bias parameter could thus be tuned to model the effects of strain, such as surface curvature, on diffusion of adatoms along the carbon-carbon bonds on zig-zag carbon nano-tubes. An interesting result in this connection (as shown in Figure 4(c) above) is that a random walk could be localized for a period of time by applying a uniform strain which favours diffusion in the direction of all bonds aligned with the tube axis.

\section{APPENDIX}

The following identites have proven useful for deriving the results in this paper

$$
\begin{aligned}
& \sinh (\gamma(b-1)) \sinh (\gamma(b-n-1))+\sinh (\gamma b) \sinh (\gamma(b-n)) \\
& =\sinh (\gamma) \sinh (\gamma(n+1))+2 \cosh (\gamma) \sinh (\gamma b) \sinh (\gamma(b-n-1)) \\
& \sinh (\gamma(b+1-n)) \sinh (\gamma b)+\sinh (\gamma(b-1)) \sinh (\gamma(b-n)) \\
& =\sinh (\gamma) \sinh (\gamma n)+2 \cosh (\gamma) \sinh (\gamma b) \sinh (\gamma(b-n)) \\
& \sinh (\gamma(b-n)) \sinh (\gamma b)+\sinh (\gamma(b-1)) \sinh (\gamma(b+1-n)) \\
& =(\cosh (\gamma(n-2))-\cosh (\gamma n)) / 2
\end{aligned}
$$

[1] E.W. Montroll and B.J. West, On an enriched collection of stochastic processes, in Fluctuation Phenomena, edited by E.W. Montroll and J.L. Lebowitz (Elsevier Science Publishers B.V., Amsterdam, 1979)

[2] R.J. Duffin, Discrete potential theory. Duke Math. J. 20, 233 (1953).

[3] P.G. Doyle and J.L. Snell, Random Walks and Electrical Networks (Mathematical Association of America, Washington D.C., (1984).

[4] O. Benichou, A.M. Cazabat, M. Moreau, and G. Oshanin, Directed random walk in adsorbed monolayer. Physica A 272, 56 (1999).

[5] R. Holyst, D. Plewczynski, A. Aksimentiev and K. Burdzy, Diffusion on curved periodic surfaces, Phys. Rev. E 60, 302 (1999). 
[6] R. Courant, K. Friedrichs, and H. Lewy, Uber die partiellen Differenzengleichungen der mathematischen Physik. Math. Ann. 100, 32 (1928).

[7] W.H. McCrea and F.J.W. Whipple, Random paths in two and three dimensions. Proc. Roy. Soc. Edinburgh. 60, 281 (1940).

[8] M.T. Batchelor and B.I. Henry, Gene Stanley, the $n$-vector model and random walks with absorbing boundaries, Physica A. 314, 77 (2002).

[9] M.T. Batchelor and B.I. Henry, Exact solution for random walks on the triangular lattice with absorbing boundaries, J. Phys. A: Math. Gen. 35, 5951 (2002).

[10] E.M. Keberle and G.L. Montet, Explicit solutions of partial difference equations and random paths on plane nets. J. Math. Anal. Appl. 6, 1 (1963).

[11] J.W. Miller, A matrix equation approach to solving recurrence relations in two-dimensional random walks. J. Appl. Prob. 31, 646 (1994).

[12] M. Ferraro and L. Zaninetti, Number of times a site is visited in two-dimensional random walks. Phys. Rev. E, 64, 056107 (2001).

[13] W. Feller, An Introduction to Probability Theory and Its Applications (Wiley, New York, 1966). p. 330 .

[14] J-L. Li, J-F. Jia, X-J. Liang, X. Liu, J-Z. Wang, Q-K Xue, Z-Q. Li, J.S. Tse, Z. Zhang and S.B. Zhang, Spontaneous assembly of perfectly ordreed identical-size nanocluster arrays, Phys. Rev. Letts. 88, 0066101 (2002)

[15] T.W. Odom, J.-L. Huang, P. Kim and C.M. Lieber, Atomic structure and electronic properties of single-walled carbon nanotubes, Nature 391(6662), 62 (1998)

[16] N. Kitamura and A. Oshiyama, Open edge growth mechanisms of single wall carbon nanotubes, J. Phys. Soc. Japan. 70, 1995 (2001)

[17] O.A. Louchev, Y. Sato and H. Kanda, Morphologiocal stabilization, destabilization, and openend closure during carbon nanotube growth mediated by surface diffusion, Phys. Rev. E 66, $011601(2002)$

[18] N. Coftas, Random walks on carbon nanotubes and quasicrystals, J. Phys. A. 33, 2917 (2000)

[19] D.J. Shu and X.G. Gong, Curvature effect on surface diffusion: The nanotube, J. Chem. Phys. 114, $10922(2001)$ 